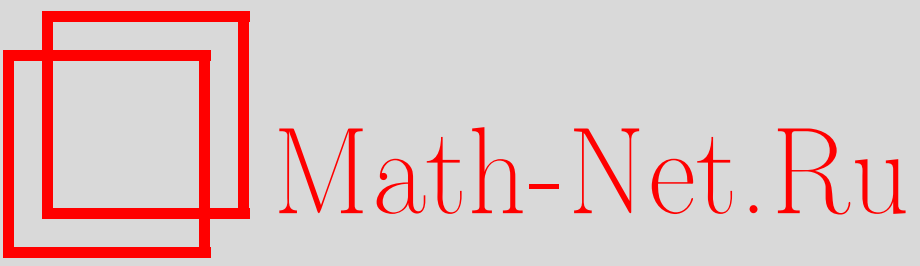

А. А. Рылов, Геометрия расслоенных графиков отображений, Итоги науки и техн. Сер. Соврем. мат. и ее прил. Темат. обз., 2020, том 179, 50-59

DOI: https://doi.org/10.36535/0233-6723-2020-179-50-59

Использование Общероссийского математического портала Math-Net.Ru подразумевает, что вы прочитали и согласны с пользовательским соглашением

http://www.mathnet.ru/rus/agreement

Параметры загрузки:

IP: 54.92 .164 .108

26 апреля 2023 г., 16:11:01 


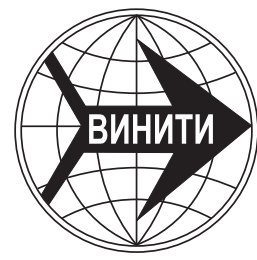

ИТОГИ НАУКИ И ТЕХНИКИ.

Современная математика и ее приложения.

Тематические обзоры.

Том 179 (2020). C. $50-59$

DOI: $10.36535 / 0233-6723-2020-179-50-59$

УДК 514.757

\title{
ГЕОМЕТРИЯ РАССЛОЕННЫХ ГРАФИКОВ ОТОБРАЖЕНИЙ
}

\author{
(c) 2020 г. $\quad$ А. А. РЫЛОВ
}

\begin{abstract}
АннотАция. В работе исследуется дифференциально-геометрический аспект отображения гладких многообразий постоянного ранга с применением понятия графика как гладкого подмногообразия в пространстве прямого произведения исходных многообразий. Случай не максимального ранга предопределяет расслоенный характер графика. Внесение римановой структуры на многообразиях обогащает геометрию графика, которая теперь существенно зависит от индуцированного поля метрического тензора: характеризуются относительно аффинные, проективные и $g$-омбилические отображения. Заключительная часть работы посвящена отображениям евклидовых пространств описанных ранее типов в терминах конструктивного графика В. Т. Базылева.
\end{abstract}

Ключевъе слова: отображение многообразий постоянного ранга, график отображения, расслоенное подмногообразие, структура почти произведения, относительно аффинное отображение, $g$-омбилическое отображение.

\section{GEOMETRY OF FIBERED GRAPHS OF MAPPINGS}

\author{
(c) 2020 A. A. RYLOV
}

\begin{abstract}
In this paper, we examine the differential-geometric aspect of constant-rank mappings of smooth manifolds based on the concept of a graph as a smooth submanifold in the space of the direct product of the original manifolds. The nonmaximality of the rank provides the fibered nature of the graph. A Riemannian structure on manifolds enriches the geometry of the graph, which now essentially depends on the induced field of the metric tensor; we characterize relatively affine, projective, and $g$-umbilical mappings. The final part of the paper is devoted to mappings of Euclidean spaces of the types described earlier in terms of V. T. Bazylev's constructive graph.
\end{abstract}

Keywords and phrases: constant-rank mapping of manifolds, graph of a mapping, fibered submanifold, almost product structure, relatively affine mapping, $g$-umbilical mapping.

AMS Subject Classification: 53A07, 53A55, 53B20

1. Введение. Геометрия отображений многообразий, снабженных различными структурами, продолжает оставаться стабильно развивающейся отраслью геометрической науки. Уже давно классикой стали монографии Н. С. Синюкова по геодезическим отображениям (см. [21]), Г. Тота по гармоническим отображениям (см. [42]). Подводя итоги многолетних исследований, коллектив исследователей, работающих в области теории отображений многообразий, снабженных дифференциально-геометрическими структурами, в 2015 г. издал книгу энциклопедического характера «Дифференциальная геометрия специальных отображений» (см. [27]) с обширной (около 1000 источников) библиографией. В настоящий момент готовится расширенное переиздание этого труда под редакцией проф. Й. Микеша. Ранее вышло несколько монографий по римановым и полуримановым субмерсиям [30,31], отдельный ряд интересных статей на эти темы (см. $[22,23,38])$. Активно развивается теория бигармонических отображений (см. $[25,33,34,36,37,43,44])$; параллельно разрабатывается глобальный аспект отображений многообразий (см. [40,41]). 
В исследованиях нередко привлекают график отображения пространств как подмногообразие в пространстве прямого произведения исходных пространств (см. $[26,29,32,39])$, в пространствах со сложной структурой вводят вспомогательные объекты типа поверхность-график (см. [10,11,28]). Классическая тематика геометрии точечных отображений отметила в 1994 г. выход монографии М. В. Драгнева, Ю. В. Павлюченко и В. В. Рыжкова [6], а в 2003 г. - монографии Г. Матиевой [14]. В последние годы в геометрии точечных отображений продолжается получение результатов (см., например, работы В. С. Болодурина [3], В. И. Грачевой [5], О. В. Казниной $[8,9]$, а также целую серию работ [15-18] под руководством Г. Матиевой). В большинстве этих работ исследование основано на привлечении конструктивного графика отображения евклидовых пространств, введенного В. Т. Базылевым (об этом $[1,2])$.

В настоящей работе будет продемонстрирован расслоенный характер графика отображения гладких многообразий постоянного, но не максимального ранга. Работа выполнена методом подвижного репера и методом внешних дифференциальных форм Картана и Лаптева (см. [7]); рассмотрения локальны, а рассматриваемые функции предполагаются достаточно гладкими.

2. Отображение гладких многообразий и его график. Пусть $M, M^{*}$ - гладкие многообразия, $\operatorname{dim} M=n, \operatorname{dim} M^{*}=m ; f: M \rightarrow M^{*}$ - гладкое отображение, определенное на некоторой области $\Omega \subset M$, так что для любого $x \in \Omega$ имеем $f(x)=y \in M^{*}$. Отображение $f$ можно интерпретировать в виде $n$-мерного гладкого подмногообразия в пространстве прямого произведения $M \times M^{*}$

$$
G=\left\{z=(x, y) \in M \times M^{*} \mid x \in \Omega ; f(x)=y \in M^{*}\right\},
$$

которое принято называть графиком отображения $f$ (см. [4, с. 56]). Пусть $\operatorname{pr}_{1}: G \rightarrow M, \operatorname{pr}_{2}: G \rightarrow$ $M^{*}$ - естественные проекции графика на каждое из многообразий. Отображение проектирования на область $\Omega \subset M$ обратимо, а обратное к нему отображение $f^{\prime}: M \rightarrow G$, определенное по закону $f(x)=z \in G$ для каждого $x \in \Omega$, можно назвать присоединенным отображением по аналогии со случаем евклидовых пространств (см. [2]).

Обозначим через $w^{J}(I, J, K=1, \ldots, n)$ и $w^{* A}(A, B, C=1, \ldots, m)$ структурные формы расслоений $H M$ и $H M^{*}$ линейных реперов $\left(\bar{e}_{I}\right)$ и $\left(\bar{e}_{A}^{*}\right)$ над заданными многообразиями. Указанные формы подчинены структурным уравнениям

$$
D \omega^{J}=\omega^{K} \wedge \omega_{K}^{J} ; \quad D \omega^{* A}=\omega^{* B} \wedge \omega_{B}^{* A} .
$$

Набор $n+m$ линейных дифференциальных форм $\omega^{J}, \omega^{* A}$ будем определять на подрасслоении таких реперов в точках графика $\left(\bar{e}_{I}, \bar{e}_{A}^{*}\right) z$, которые соответствующими линейными отображениями $\operatorname{Pr}_{1}$ и $\operatorname{Pr}_{2}$ проектируются в подвижные реперы $\left(\bar{e}_{I}\right)_{x}$ и $\left(\bar{e}_{A}^{*}\right)_{y}$. Структурные уравнения отображения $f$ (см. [13]) имеют вид

$$
\omega^{* A}=h_{I}^{A} \omega^{I} .
$$

Продолжая эту систему, согласно уравнениям (1) получаем

$$
\Delta h_{I}^{A}=h_{I J}^{A} \omega^{J}, \quad h_{I J}^{A}=h_{J I}^{A},
$$

где $\Delta h_{I}^{A}:=d h_{I}^{A}-h_{K}^{A} \omega_{I}^{K}+h_{I}^{B} \omega_{B}^{* A}$, а линейные дифференциальные формы $\omega_{I}^{J}$ и $\omega_{B}^{* A}$ определены на подрасслоении второго порядка $H^{2} G$. Величины $\left\{h_{I}^{A}\right\}$ и $\left\{h_{I}^{A}, h_{I J}^{A}\right\}$ являются относительными компонентами фундаментальных объектов первого и второго порядка, порожденных отображением(см. [13, с. 40]). С помощью объекта $\left\{h_{I}^{A}\right\}$ определяются координаты $X^{* A}$ образа $f_{*} \bar{e}$ векторного поля $\bar{e}$ в дифференциале отображения $f_{*}: X^{* A}=h_{I}^{A} X^{I}$. В настоящей работе изучаются гладкие отображения $f: M \rightarrow M^{*}$ с постоянным на области $\Omega \subset M$ положительным, но не максимальным рангом $r$, где $0<r<\min (n, m)$. Это означает, что для всякого координатного представления $y^{A}=y^{A}\left(x^{1}, \ldots, x^{n}\right)$ отображения $f$ ранг якобиевой матрицы $\partial y^{A} / \partial x^{I}$ равен числу $r$. Значит, $\operatorname{rank}\left(h_{I}^{A}\right)=r$ во всех точках области $\Omega$. Проведем дальнейшую канонизацию репера $\left(\bar{e}_{I}, \bar{e}_{A}^{*}\right)_{z}$ :

(а) в соответствующем репере $\left(\bar{e}_{I}\right)_{x}$ последние $(n-r)$ векторов $\bar{e}_{\alpha}(\alpha, \beta, \gamma=r+1, \ldots, n)$ образуют базис ядра $\operatorname{Ker} f_{* x}$ в каждой точке $x \in \Omega$, а первые $r$ векторов $\bar{e}_{i}(i, j, k, l, s, t=1, \ldots, r)$ дополняют $\bar{e}_{\alpha}$ до базиса всего касательного пространства $T_{x} M$; 
(b) в соответствующем репере $\left(\bar{e}_{A}^{*}\right)_{y}$ первые $r$ векторов $\bar{e}_{i}^{*}=f_{* x}\left(\bar{e}_{i}\right)$ составляют базис образа $\operatorname{Im} f_{* x}$, а остальные векторы $\bar{e}_{a}^{*}(a, b, c=r+1, \ldots, m)$ дополняют $\bar{e}_{i}^{*}$ до базиса всего пространства $T_{y} M^{*}$. Этой канонизации репера отвечает приведение матрицы $h_{I}^{A}$ к диагональному виду $\left(\begin{array}{cc}\delta_{i}^{j} & 0 \\ 0 & 0\end{array}\right)$ и сле-
дующей записи структурных уравнений $(2)$ отображения:

$$
\omega^{* i}=\omega^{i}, \quad \omega^{* a}=0 .
$$

Продолжение этих уравнений с учетом (3) дает следующий набор соотношений:

$$
\begin{gathered}
\omega_{i}^{* j}-\omega_{i}^{j}=h_{i k}^{j} \omega^{k}+h_{i \alpha}^{j} \omega^{\alpha}, \\
\omega_{i}^{* a}=h_{i k}^{a} \omega^{k}, \\
-\omega_{\alpha}^{j}=h_{\alpha k}^{j} \omega^{k}+h_{\alpha \beta}^{j} \omega^{\beta}, \\
h_{\alpha k}^{b}=0, \quad h_{\alpha \gamma}^{b}=0 .
\end{gathered}
$$

Теорема 1. Гладкое отображение многообразий $f: M \rightarrow M^{*}$, заданное на некоторой области $\Omega \subset M$, имеет постоянный ранг $r$ тогда и только тогда, когда график отображсения $G \subset M \times M^{*}$ представляет собой расслоенное подмногообразие над $r$-мерным образом $f(\Omega) \subset$ $M^{*}$.

Доказательство. Пусть отображение $f: M \rightarrow M^{*}$, заданное на области $\Omega \subset M$, имеет постоянный ранг $r$. Тогда при описанной выше канонизации реперов на области, на ее образе $f(\Omega) \subset M^{*}$ и на графике $G \subset M \times M^{*}$ имеют место уравнения (4)-(7). Значит, подсистема форм $\omega^{k}$ определяет на графике некоторое интегрируемое $(n-r)$-распределение, соответствующее распределению Ker $f_{*}$ элементов ядра дифференциала $f_{*}$.

Обозначим через $F_{x}$ максимальное интегральное многообразие распределения $\operatorname{Ker} f_{*}$, т.е. $F_{x}=f^{-1}(y)$, а через $F_{z}^{\prime}=\left(F_{x}, y\right)-(n-r)$-мерное интегральное многообразие распределения $\Delta_{\nu}$ на графике $G$, которое можно называть вертикальным. Согласно уравнениям (4) и (6) система

$$
\omega^{* i}=\omega^{i}, \quad \omega^{* a}=0, \quad \omega_{i}^{* a}=h_{i k}^{a} \omega^{k}
$$

определяет $r$-мерное подмногообразие $f(\Omega) \subset M^{*}$, а полный образ $\operatorname{Im} f_{*}$, определяемый базисом $\bar{e}_{i}^{*}$, представляет собой касательное расслоение над многообразием $f(\Omega)$. Субмерсия $\operatorname{pr}_{2}: G \rightarrow f(\Omega)$ выполняет роль канонической проекции, расслаивающей график $G$ на $(n-r)$ мерные слои $F^{\prime}$ над базой $f(\Omega)$. В силу соотношений $(1),(4)-(5)$ формы $\omega^{i}$-базовые, $\omega^{\alpha}-$ слоевые; они удовлетворяют структурным уравнениям расслоенного пространства:

$$
D \omega^{i}=\omega^{k} \wedge \omega_{k}^{* i} ; \quad D \omega^{\alpha}=\omega^{k} \wedge \omega_{k}^{\alpha}+\omega^{\beta} \wedge \omega_{\beta}^{\alpha} .
$$

Обратно, пусть теперь $f: M \rightarrow M^{*}$-гладкое отображение, определенное на области $\Omega \subset M$, $\operatorname{dim} M=n, \operatorname{dim} M^{*}=m$. Предположим, что его $n$-мерный график представляет собой расслоенное подмногообразие $G \subseteq M \times M^{*}$ над $r$-мерным образом $f(\Omega) \subset M^{*}$. Очевидно, что $r<m$. Каноническая проекция $\mathrm{pr}_{2}: G \rightarrow f(\Omega)$ расслаивает график на $r$-параметрическое семейство $(n-r)$ мерных многообразий $F^{\prime}$, причем во всякой точке $z \in G$ имеем $F_{z}^{\prime}=\left\{\left(F_{x}, y\right) \mid F_{x}=f^{-1}(y)\right\}$. Выберем репер $\left(\bar{e}_{i}, \bar{e}_{\alpha}\right)_{x}$, в котором векторы $\bar{e}_{\alpha}$ - базис касательного пространства $T_{x} F_{x}$, а для соответствующего репера $\left(\bar{e}_{i}^{*}, \bar{e}_{a}^{*}\right)_{y}$ векторы $\bar{e}_{i}^{*}=f_{* x}\left(\bar{e}_{i}\right)$ - базис пространства $T_{y} f(\Omega)$. Тогда $f_{* x}\left(\bar{e}_{\alpha}\right)=\bar{e}$ и в области $\Omega \subset M$ имеем $\operatorname{rank}\left(h_{I}^{A}\right)=r<\min (n, m)$, т. е. отображение $f$ имеет пониженный ранг $r$.

3. Риманова структура графика отображения. Пусть $(M, g),\left(M^{*}, g^{*}\right)$ - римановы многообразия, где $g, g^{*}$ обозначают соответствующее поле метрического тензора на гладком многообразии; $\operatorname{dim} M=n, \operatorname{dim} M^{*}=m$. Обозначим через $\left(w^{I}, \omega_{J}^{I}\right)$ и $\left(w^{* A}, \omega_{B}^{* A}\right)$ формы римановых 
связностей на $M$ и $M^{*}$, удовлетворяющие структурным уравнениям

$$
\begin{gathered}
D \omega^{I}=\omega^{J} \wedge \omega_{J}^{I} ; \quad D \omega_{J}^{I}=\omega_{J}^{K} \wedge \omega_{K}^{I}+R_{J K L}^{I} \omega^{K} \wedge \omega^{L} ; \\
d g_{I J}-g_{I K} \omega_{J}^{K}-g_{K J} \omega_{I}^{K}=0 ; \\
D \omega^{* A}=\omega^{* B} \wedge \omega_{B}^{* A} ; \quad D \omega_{B}^{* A}=\omega_{B}^{* C} \wedge \omega_{C}^{* A}+R_{B C D}^{* A} \omega^{* C} \wedge \omega^{* D} ; \\
d g_{A B}^{*}-g_{A C}^{*} \omega_{B}^{* C}-g_{C B}^{*} \omega_{A}^{* C}=0 ;
\end{gathered}
$$

где $R_{J K L}^{I}, R_{B C D}^{* A}$ - тензоры кривизны связностей Леви-Чивиты соответствующих римановых многообразий $M$ и $M^{*}$. На пространстве прямого произведения $M \times M^{*}$ определяется риманова структура полем метрического тензора $g \oplus g^{*}$ с компонентами

$$
\left(\begin{array}{cc}
g_{I J} & 0 \\
0 & g_{A B}^{*}
\end{array}\right)
$$

Если задано гладкое отображение $f:(M, g) \rightarrow\left(M^{*}, g^{*}\right)$ на некоторой области $\Omega \subset M$ так, что $\forall x \in \Omega: f(x)=y \in M^{*}$, то график отображения - это $n$-мерное подмногообразие $G \subset$ $M \times M^{*}$ в римановом пространстве. Дальнейшее продолжение структурных уравнений (2)-(3) отображения $f$ приводит к системе

$$
\Delta h_{I J}^{A}=h_{I J K}^{A} \omega^{K}
$$

где $\Delta h_{I J}^{A}:=d h_{I J}^{A}-h_{I L}^{A} \omega_{J}^{L}-h_{L J}^{A} \omega_{I}^{L}+h_{I J}^{B} \omega_{B}^{* A}, h_{I[J K]}^{A}=h_{L}^{A} R_{I J K}^{L}-h_{I}^{B} h_{J}^{C} h_{K}^{D} R_{B C D}^{* A}$. Теперь система величин $h_{I J}^{A}$ образует тензор на графике, носящий название второго (фундаментального) тензоpa отображения $f$. Его компоненты определяют координатную запись билинейного отображения $\sigma: T M \times T M \rightarrow T f(M)$, называемого второй фундаментальной формой отображения $f$ (см. [35]). Отображение $\sigma$ всякой паре векторных полей $\bar{e}$ и $\bar{e}$ ставит в соответствие векторное поле на образе $f(\Omega)$ по закону: $\sigma(\bar{e}, \bar{e})=h_{I J}^{A} X^{I} Y^{J} \bar{e}_{A}^{*}$.

Следствие 1.1. Гладкое отображение римановых многообразий $f:(M, g) \rightarrow\left(M^{*}, g^{*}\right)$ nониженного ранга $r$, заданное на некоторой области $\Omega \subset M$, порождает на ней полуинтегрируемую структуру почти произведения из вертикального слоения Ker $f_{*}$ элементов ядра дифберенциала отображения $f_{*}$ и ортогонального ему $r$-мерного распределения.

Доказательство. Для обоснования утверждения уточним канонизацию репера $\left(\bar{e}_{i}, \bar{e}_{\alpha}\right)_{x}$ из доказательства Теоремы 1, выбрав $\left(\bar{e}_{\alpha}\right)$ - ортонормированным базисом касательного пространства $T_{x} F_{x}$, а $\left(\bar{e}_{i}\right)$ - ортогональными элементу ядра дифференциала отображения $f_{*}$. В свою очередь векторы $\left(\bar{e}_{a}^{*}\right)$ репера $\left(\bar{e}_{i}^{*}, \bar{e}_{a}^{*}\right)_{y}$ составляют ортонормированный базис ортогонального дополнения подпространства $\operatorname{Im} f_{*}$ до пространства $T_{y} f(\Omega)$. Тогда в подрасслоении $H_{0} G$ линейных реперов графика компоненты метрических тензоров приведены к виду

$$
g=\left(\begin{array}{cc}
g_{i j} & 0 \\
0 & \delta_{\alpha \beta}
\end{array}\right), \quad g^{*}=\left(\begin{array}{cc}
g_{i j}^{*} & 0 \\
0 & \delta_{a b}
\end{array}\right), \quad \widetilde{g}=\left(\begin{array}{cc}
g_{i j}^{*} & 0 \\
0 & 0
\end{array}\right)
$$

Не выписывая полного набора структурных уравнений для метрических тензоров, обратим внимание на одно из них:

которое с учетом формул (7) примет вид

$$
\omega_{i}^{\beta}=-g_{i k} \omega_{\beta}^{k},
$$

$$
\omega_{i}^{\beta}=h_{i j \beta} \omega^{j}+h_{i \gamma \beta} \omega^{\gamma},
$$

где $h_{i j \beta}:=g_{i k} h_{j \beta}^{k} ; h_{i \gamma \beta}:=g_{i k} h_{\gamma \beta}^{k}$. Эта система представляет собой структурные уравнения распределения $\Delta r$-мерных плоскостей, в каждой точке $x \in \Omega$ ортогональных слоям $F_{x}=f^{-1}(y)$. Таким образом, на области $\Omega \subset M$ задана полуинтегрируемая структура почти произведения

$$
T M=\Delta \oplus \operatorname{Ker} f_{*},
$$

где $\Delta=\operatorname{Ker}^{\perp} f_{*}$.

Тождественное равенство нулю второй фундаментальной формы отображения $f: h_{I J}^{A}=0$ характеризует тип афбциных, или вполне геодезических отображений (см. [45]). Они отображают 
геодезические кривые области $\Omega \subset M$ в геодезические многообразия $M^{*}$ с сохранением аффинного параметра кривой.

Если поле ненулевого второго тензора отображения $f$ допускает представление с помощью компонент $h_{I}^{A}$ первого тензора отображения

$$
h_{I J}^{A}=a_{I} h_{J}^{A}+a_{J} h_{I}^{A},
$$

где $a_{I}$ - компоненты некоторого ковектора на графике $G$, то $f$ отображает геодезические области $\Omega \subset M$ в геодезические многообразия $M^{*}$ без сохранения аффинного параметра и носит название проективного отображения.

Отображение $f:(M, g) \rightarrow\left(M^{*}, g^{*}\right)$ индуцирует на области $\Omega$ поле тензора с компонентами $\widetilde{g}$

$$
\widetilde{g}_{I J}=h_{I}^{A} h_{J}^{B} g_{A B}^{*}
$$

и соответствующую метрику $d \widetilde{s}^{2}=\widetilde{g}_{I J} \omega^{I} \omega^{J}$. Выделим ряд известных типов отображений римановых многообразий, существенно использующих наличие метрики. Отображение $f:(M, g) \rightarrow$ $\left(M^{*}, g^{*}\right)$ называют относительно аффинным (см. [46]), если оно индуцирует на области $\Omega \subset M$ поле тензора $\widetilde{g}$, ковариантно постоянного относительно римановой связности на области $\Omega$ :

$$
\nabla \widetilde{g}_{I J}=0 .
$$

Согласно уравнениям структуры тензорного поля и циклической замене индексов приходим к следствию:

$$
h_{I}^{A} h_{J K}^{B} g_{A B}^{*}=0 .
$$

Таким образом, всякое аффинное отображение является относительно аффинным. Известно (см. [46]), что относительно аффинное отображение связного многообразия в другое многообразие имеет постоянный ранг.

С другой стороны, поле второго тензора отображения всегда допускает представление в виде суммы

$$
h_{I J}^{A}=\xi^{A} g_{I J}+h_{I J}^{\circ A},
$$

где $\xi^{A}=\frac{1}{n} g^{I J} h_{I J}^{A}, h_{I J}^{\circ A}$ - бесследовая часть. Величины $h^{A}=g^{I J} h_{I J}^{A}$ подчиняются структурным уравнениям

$$
d h^{A}+h^{B} \omega_{B}^{* A}=g^{I J} h_{I J K}^{A} \omega^{* K},
$$

поэтому функции $h^{A}$ являются компонентами векторного поля на образе $f(\Omega)$, носящего название поля напряжения отображения $f$.

В соответствии с представлением второго тензора $h_{I J}^{A}$ можно выделить два класса отображений (а) первый класс характеризуется нулевым полем напряжения:

$$
g^{I J} h_{I J}^{A}=0
$$

состоит из гармонических отображений;

(b) второй класс выделяется нулевой бесследовой частью разложения:

$$
h_{I J}^{A}=\xi^{A} g_{I J},
$$

такие отображения будем называть $g$-омбилическими. Заметим, что отображение $f:(M, g) \rightarrow$ $\left(M^{*}, g^{*}\right)$ является аффинным тогда и только тогда, когда оно одновременно $g$-омбилическое и гармоническое: $h_{I J}^{A}=0$.

Если вторая фундаментальная форма отображения $f$ пропорциональна метрике $d \widetilde{s}^{2}$, то отображение будем называть $g^{*}$-омбилическим.

Из соотношений (12)-(16) на компоненты первого и второго тензора отображения в силу проведенной канонизации реперов непосредственно следует

Предложение 1 (см. [20]). Если гладкое отображсние римановых многообразий $f:(M, g) \rightarrow$ $\left(M^{*}, g^{*}\right)$, заданное на некоторой области $\Omega \subset M$, постоянного ранга $r$ является

(1) относительно аффинным, то вертикальное слоение $\operatorname{Ker} f_{*}$ вполне геодезическое, а распределение $\Delta=\operatorname{Ker}^{\perp} f_{*}$ задает также вполне геодезическое слоение; 
(2) проективным, то вертикальное слоение $\operatorname{Ker} f_{*}$ вполне геодезическое, а распределение $\Delta=$ $\operatorname{Ker}^{\perp} f_{*}$ задает омбилическое слоение;

(3) g-омбилическим $(1<r<n-1)$, то вертикальное слоение $\operatorname{Ker} f_{*}$ омбилическое, а распределение $\Delta=\mathrm{Ker}^{\perp} f_{*}$ задает вполне геодезическое слоение.

Перейдем от подвижного репера $\left(\bar{e}_{I}, \bar{e}_{A}^{*}\right)_{z}$ к новому реперу $\left(\bar{e}_{I}, \bar{e}_{A}^{*}\right)_{z}$, в котором

(a) векторы $\bar{e}_{I}=\bar{e}_{I}+h_{I}^{A} \bar{e}_{A}^{*}$ образуют базис касательного пространства $T_{z} G$ к графику $f$,

(b) векторы $\bar{e}_{A}^{*}$ составляют базис ортогонального дополнения $T_{z}^{\perp} G$ касательного пространства до всего прямого произведения $M \times M^{*}$.

Тем самым подрасслоение указанных реперов над многообразием $G$ допускает представление в виде суммы $H_{0}^{\prime} G \oplus H_{0}^{\perp} G$, где $H_{0}^{\prime} G$ - подрасслоение реперов $\left(\bar{e}_{I}\right), H_{0}^{\perp} G$ - подрасслоение нормальных реперов $\left(\bar{e}_{A}^{*}\right)$. Как было отмечено выше, отображение $f:(M, g) \rightarrow\left(M^{*}, g^{*}\right)$ индуцирует на области $\Omega$ поле тензора $\tilde{g}$ и соответствующую метрику $\tilde{d}^{2}$. Тем временем на графике индуцируется поле тензора с компонентами

$$
G_{I J}=\left\langle\bar{e}_{I}, \bar{e}_{J}\right\rangle=g_{I J}+\tilde{g}_{I J}
$$

и метрика $d S^{2}=G_{I J} \omega^{I} \omega^{J}$. Введя обозначение $G_{A B}^{*}=\left\langle\bar{e}_{A}^{*}, \bar{e}_{B}^{*}\right\rangle$, получаем метрику пространства $M \times M^{*}$ в виде

$$
\left(\begin{array}{cc}
G_{I J} & 0 \\
0 & G_{A B}^{*}
\end{array}\right)
$$

Для риманова многообразия $M \times M^{*}$ можно выписать структурные уравнения вида (8), формы римановой связности в них будем обозначать заглавными буквами $\left(\Omega_{J}^{I}, \Omega_{I}^{n+B}, \Omega_{n+A}^{J}, \Omega_{n+A}^{n+B}\right)$. В подвижном репере $\left(\bar{e}_{I}, \bar{e}_{A}^{*}\right)_{z}$ структурные уравнения подмногообразия $G \subset M \times M^{*}$ записываются в виде

$$
\Omega^{n+A}=0,
$$

где $\Omega^{n+A}:=\omega^{* A}-h_{I}^{A} \omega^{I}$. Продолжение этой системы дает соотношения

$$
\Omega_{I}^{n+A}=t_{I J}^{n+A} \omega^{J}, \quad t_{I J}^{n+A}=t_{J}^{n+A},
$$

в которых через $t_{I J}^{n+A}$ обозначены относительные компоненты второго фундаментального тензора графика $G$. Из нового выражения метрики пространства $M \times M^{*}$ следует справедливость равенства

$$
G_{I J} \Omega_{n+A}^{J}+G_{A B}^{*} \Omega_{I}^{n+B}=0,
$$

откуда имеем $\Omega_{n+A}^{J}=-G^{I J} G_{A B}^{*} \Omega_{I}^{n+B}$. Исходя из этих соотношений, уравнения структуры подрасслоений $H_{0}^{\prime} G$ и $H_{0}^{\perp} G$ приводятся к виду

$$
\begin{gathered}
D \omega^{I}=\omega^{J} \wedge \omega_{J}^{I} ; \\
D \Omega_{I}^{J}=\Omega_{I}^{K} \wedge \Omega_{K}^{J}+\tilde{R}_{I K L}^{J} \omega^{K} \wedge \omega^{L} ; \\
D \Omega_{n+A}^{n+B}=\Omega_{n+A}^{n+C} \wedge \Omega_{n+C}^{n+B}+\tilde{R}_{A K L}^{B} \omega^{K} \wedge \omega^{L},
\end{gathered}
$$

где $\tilde{R}_{I K L}^{J}:=-G^{J S} G_{A B}^{*} t_{I[K}^{n+A} t_{L] S}^{n+B} ; \tilde{R}_{A K L}^{B}:=-G_{A C}^{*} G^{I J} t_{I[K}^{n+C} t_{L] J}^{n+B}$. В силу теоремы Картана-Лаптева на указанных расслоениях реперов определены две связности:

(а) первая - с формами $\Omega_{I}^{J}$ и тензором кривизны $\tilde{R}_{I K L}^{J}$, так называемая индуцированная связность Леви-Чивиты на графике $G \subset M \times M^{*}$;

(b) вторая - нормальная свлзность (см., например, [12, т. II, с. 23]) графика $G$ с формами $\Omega_{n+A}^{n+B}$ и тензором кривизны $\tilde{R}_{A K L}^{B}$. 
4. Конструктивный график отображения евклидовых пространств. Пусть $M=E^{n}$, $M^{*}=\bar{E}^{m}$ - два евклидовых пространства; $f: E^{n} \rightarrow \bar{E}^{m}$ - гладкое отображение, определенное на некоторой области $\Omega \subset E^{n}$ так, что $\forall x \in \Omega: f(x)=y \in \bar{E}^{m}$, и имеющее постоянный ранг $r$. Следуя В. Т. Базылеву (см. [2]), вложим пространства $E^{n}$ и $\bar{E}^{m}$ как вполне ортогональные плоскости с общей точкой $O$ в евклидово пространство $E^{n+m}$. В этом объемлющем пространстве гладкому отображению $f$ соответствует $n$-мерная поверхность

$$
G=\left\{z \in E^{n+m} \mid \overrightarrow{O z}=\overrightarrow{O x}+\overrightarrow{O y} ; x \in \Omega ; f(x)=y \in \bar{E}^{m}\right\}
$$

называемая конструктивным графиком отображения $f$ (см. также [1]).

Слои отображения, т. е. максимальные интегральные многообразия распределения ядра $\operatorname{Ker} f_{*}$, в этом случае принято называть горизонталями отображения $f$ (см. [2]). Если $F_{x}$ - горизонталь отображения, проходящая через точку $x$, то $f\left(F_{x}\right)=y$. Проведем через точку $y$ плоскость $E^{n}(y)$, параллельную $E^{n}$, тогда $(n-r)$-мерная поверхность $F_{z}^{\prime}=G \cap E^{n}(y)$ проектируется вдоль плоскости $\bar{E}^{m}$ в горизонталь $F_{x}$ и называется горизонтальным сечением графика отображения $f$ в точке $z$. Поскольку присоединенное отображение $f^{\prime}: E^{n} \rightarrow G$ сводится к параллельному переносу, и $F_{z}^{\prime} \cong F_{x}$, то график $G$ частично параллелен плоскости $E^{n}$ с индексом $1-\frac{r}{n}$. Итак, график $G \subset E^{n+m}$ отображения $f: E^{n} \rightarrow \bar{E}^{m}$ евклидовых пространств ранга $r$ расслаивается на $r$-параметрическое семейство горизонтальных сечений, каждое из которых проектируется вдоль плоскости $\bar{E}^{m}$ в соответствующую горизонталь отображения $f$. Деривационные формулы репера в точке $z \in G$ графика могут быть записаны следующим образом:

$$
d \bar{z}=\omega^{i} \bar{e}_{i}+\omega^{\alpha} \bar{e}_{\alpha} ; \quad d \bar{e}_{I}=\Omega_{I}^{J} \bar{e}_{J}+\Omega_{I}^{n+A_{\bar{e}}} \bar{e}_{A}^{*} ; \quad d \bar{e}_{A}^{*}=\Omega_{n+A}^{J} \bar{e}_{J}+\Omega_{n+A}^{n+B} \bar{e}_{B}^{*} .
$$

Заметим, что в каждой точке графика векторы $\bar{e}_{A}^{*}$ репера инвариантно определяют две ортогонально дополнительные плоскости: одну из них $\left[z, \bar{e}_{i}^{*}\right]$ назовем ранговой нормалью, а другую $\left[z, \bar{e}_{a}^{*}\right]$ - перенесенной нормалью.

Компоненты второго тензора графика $G$ выражаются через координаты $h_{I J}^{A}$ второго тензора отображения $f$ следующим образом:

$$
t_{I J}^{n+l}=-G^{l k} g_{k j}^{*} h_{I J}^{j}, \quad t_{i j}^{n+a}=h_{i j}^{a}, \quad t_{i \beta}^{n+a}=t_{\alpha \beta}^{n+a}=0 .
$$

Получение характеристик графика $G$ отображения евклидовых пространств будем рассматривать для таких отображений $f$ ранга $r>1$, у которых присоединенное отображение $f^{\prime}: E^{n} \rightarrow G \subset$ $E^{n+m}$ является nростым (см. [19]). Это означает, что интегральные кривые полей главных направлений эллипсоидов деформации отображения $f^{\prime}$ однозначно определяют на области $\Omega \subset E^{n}$ ортогональную сеть $\sigma^{n}$, называемую основанием отображения; при этом сеть $\sigma^{\prime n}=f^{\prime}\left(\sigma^{n}\right)$ на графике $G$ также ортогональна.

Теорема 2. Пусть гладкое отображение евклидовых пространств $f: E^{n} \rightarrow \bar{E}^{m}$, заданное на некоторой области $\Omega \subset E^{n}$, с графиком $G \subset E^{n+m}$ имеет постолнный ранг $r$. Справедливы следующие утверждения.

1. Если $f$ является относительно аффинным, то его график лежит на иилиндрической поверхности, образующими которой служат $(n-r)$-мерные плоские горизонтальные сечения, а направляющая, проходящая через точку $z \in G$, имеет $m$-мерную соприкасающуюся плоскость; обратное также верно.

2. Если $f$ является проективным, то его график расслаивается на два семейства поверхностей: $(n-r)$-мерных плоских горизонтальных сечений и перпендикулярных им r-ортогональных систем.

3. Если $f$ является g-омбилическим, то его график расслаивается на два семейства поверхностей: $(n-r)$-мерные горизонтальные сечения являются омбилическими подмногообразиями пространства $E^{n+m}$, а ортогональные им поверхности другого семейства несут голономную сеть линий кривизнь.

4. Если $f$ является $g^{*}$-омбилическим, то его график лежит на иилиндрической поверхности, образующими которой слуюат $(n-r)$-мерные плоские горизонтальные сечения, а направляющие суть r-мерные поверхности, несущие голономную сеть линий кривизны. 
Доказательство. Пусть отображение евклидовых пространств $f: E^{n} \rightarrow \bar{E}^{m}$ на области $\Omega \subset E^{n}$ имеет постоянный ранг $r$.

1. Если $f$ - относительно аффинное, то согласно условию (14) и в силу соотношений (19) имеем

$$
\begin{gathered}
\Omega_{i}^{\alpha}=h_{i \alpha J} \omega^{J}=0 ; \quad \Omega_{i}^{n+k}=t_{i J}^{n+k} \omega^{J}=0 ; \\
\Omega_{\alpha}^{j}=g_{k l} g^{* l j} \Omega_{\alpha}^{n+k}=0 ; \quad \Omega_{\alpha}^{n+a}=0 .
\end{gathered}
$$

Таким образом, в формулах (18) $d \bar{e}_{i}=\Omega_{i}^{j} \bar{e}_{j}+h_{i j}^{a} \omega^{j} \bar{e}_{a}^{*} ; d \bar{e}_{\alpha}=\Omega_{\alpha}^{\beta} \bar{e}_{\beta}$.

Следовательно, график $G$ отображения локально представляет собой цилиндрическую поверхность, $(n-r)$-мерными плоскими образующими которой являются горизонтальные сечения. Направляющей этой поверхности служит $r$-мерное подмногообразие $F_{z}^{\prime \perp}$ - образ максимального вполне геодезического интегрального многообразия $F_{x}^{\perp}$ распределения $\Delta$ в присоединенном отображении $f^{\prime}: E^{n} \rightarrow G$. При этом соприкасающаяся плоскость указанной направляющей в точке $z \in G$

$$
\Pi_{z}\left(F_{z}^{\prime \perp}\right)=\left[z, d \bar{z}, d^{2} \bar{z}\right]=\left[z, \omega^{i} \bar{e}_{i},\left(d \omega^{i}+\omega^{j} \Omega_{j}^{i}\right) \bar{e}_{i}+h_{i j}^{a} \omega^{i} \omega^{j} \bar{e}_{a}^{*}\right]
$$

является $m$-мерной, а главная нормаль подмногообразия $F_{z}^{\prime \perp}$ в точке графика совпадает с перенесенной нормалью $\left[z, \bar{e}_{a}^{*}\right]$.

Отметим важный частный случай: график аффинного отображения $f: E^{n} \rightarrow \bar{E}^{m}$ лежит на $n$ мерной плоскости (верно и обратное). Для обоснования утверждений $2-4$ уточним канонизацию репера $\left(\bar{e}_{i}, \bar{e}_{\alpha}\right)_{x}$ на области $\Omega \subset E^{n}$, расположив его векторы на касательных к линиям $\sigma^{n}$ основания присоединенного отображения. Интегральные кривые полей векторов $\left(\bar{e}_{i}\right)$ образуют на области $\Omega \subset E^{n} r$-мерную ортогональную подсеть $\sigma^{r} \subset \sigma^{n}$, которой на графике $G$ соответствует ортогональная подсеть $f^{\prime}\left(\sigma^{r}\right)=\sigma^{\prime r} \subset \sigma^{\prime n}=f^{\prime}\left(\sigma^{n}\right)$. Условие голономности (см. [1]) указанных выше подсетей $\sigma^{r}$ и $\sigma^{\prime r}$ означает равенство нулю координат $h_{i j}^{k}=0$ для различных значений $i, j, k$.

2. Если $f$ - проективное, то согласно условию (12) и в силу соотношений (19) имеем

$$
h_{i k}^{j}=0(j \neq i, k) ; \quad h_{i \alpha}^{j}=0 ; \quad \Omega_{i}^{n+a}=0 ; \quad \Omega_{\alpha}^{j}=0 ; \quad \Omega_{\alpha}^{n+A}=0 .
$$

Таким образом, в формулах (18) $d \bar{e}_{\alpha}=\Omega_{\alpha}^{\beta} \bar{e}_{\beta}$. Следовательно, график $G$ отображения расслаивается на два семейства ортогональных поверхностей: $(n-r)$-мерных плоских горизонтальных сечений и $r$-мерных поверхностей, несущих ортогональную голономную сеть(т. е. так называемых $r$-ортогональных систем).

3. Если $f-g$-омбилическое, то согласно условию (16) и в силу соотношений (19) имеем

$$
\begin{gathered}
h_{i j}^{k}=0, h_{i j}^{a}=0(i \neq j) \\
\Omega_{\alpha}^{k}=\frac{1}{g_{k k}^{*}} \Omega_{\alpha}^{n+k}=-\frac{1}{1+g_{k k}^{*}} \xi^{k} \omega^{\alpha}, \text { по } k \text { нет суммирования. }
\end{gathered}
$$

Таким образом, график $G$ отображения расслаивается на два семейства ортогональных поверхностей: $(n-r)$-мерных горизонтальных сечений, которые являются омбилическими подмногообразиями (см. [24]) пространства $E^{n+m}$, и $r$-мерных поверхностей, несущих ортогональную голономную сеть $\sigma^{\prime r} \subset \sigma^{\prime n}$. Более того, условия

$$
t_{i j}^{n+k}=t_{i j}^{n+a}=0(i \neq j) ; h_{i j \alpha}=0
$$

имеют следствием сопряженность указанной сети. Значит, $\sigma^{\prime r}$ - голономная сеть линий кривизны.

4. Если $f-g^{*}$-омбилическое, то в силу соотношений (19) имеем

$$
\begin{aligned}
h_{i j}^{k} & =0, h_{i j}^{a}=0(i \neq j) ; \\
\Omega_{i}^{\alpha}=h_{i \alpha J} \omega^{J}=0 ; \quad \Omega_{i}^{n+k} & =-\frac{g_{k k}^{*}}{1+g_{k k}^{*}} \eta^{k} \omega^{i}, \text { по } k \text { нет суммирования; } \\
\Omega_{\alpha}^{j} & =0 ; \quad \Omega_{\alpha}^{n+A}=0 .
\end{aligned}
$$

Таким образом, в формулах (18) $d \bar{e}_{i}=\Omega_{i}^{j} \bar{e}_{j}+\eta^{a} \omega^{i} \bar{e}_{a}^{*} ; d \bar{e}_{a}=\Omega_{\alpha}^{\beta} \bar{e}_{\beta}$. 
Поэтому график $G$ отображения локально представляет собой цилиндрическую поверхность, $(n-r)$-мерными плоскими образующими которой являются горизонтальные сечения, а направляющей этой поверхности служит $r$-мерное подмногообразие $F_{z}^{\prime \perp}$, по аналогии со случаем 3 несущее голономную сеть линий кривизны.

\section{СПИСОК ЛИТЕРАТУРЫ}

1. Базылев В. Т. К геометрии дифференцируемых отображений евклидовых пространств// Уч. зап. МПГУ им. В. И. Ленина. - 1970. - № 374. - С. 41-51.

2. Базылев В. Т. О частичных отображениях евклидовых пространств// Тр. Мат. ин-та им. А. Размадзе АН ГрузССР. - 1980. - 64. - C. 19-27.

3. Болодурин В. С. О свойствах точечных соответствий между тремя многомерными поверхностями проективных пространств// Изв. вузов. Мат. - 2013. - № 9. - С. 3-15.

4. Бурбаки Н. Дифференцируемые и аналитические многообразия. Сводка результатов. - М.: Мир, 1975.

5. Грачева В. И. О геометрии точечного соответствия евклидовых пространств при использовании графика дифференцируемого отображения// в кн.: Математический вестник педагогических вузов и университетов Волго-Вятского региона. - Киров: Радуга-ПРЕСС, 2012. - С. 90-97.

6. Драгнев М. В., Павлюченко Ю. В., Рыжков В. В. Избранные главы дифференциальной геометрии точечных отображений. - М.: РУДН, 1994.

7. Евтушик Л. Е., Лумисте Ю. Г., Остиану Н. М., Широков А. П. Дифференциально-геометрические структуры на многообразиях// Итоги науки и техн. Пробл. геом. $-1979 .-9 .-$ С. 3-247.

8. Казнина О. В. О графике некоторого отображения в конструкции Фубини-Чеха// в кн.: Математика в образовании. - Чебоксары, 2011. - С. 176-180.

9. Казнина О. В. Типы отображения в конструкции Фубини-Чеха// в кн.: Математический вестник педагогических вузов и университетов Волго-Вятского региона. - Киров: Радуга-ПРЕСС, 2013. C. $86-91$.

10. Карманова М. Б. Поверхности-графики над трехмерными пространствами Карно-Каратеодори// Докл. РАН - 2015. - 463, № 3. - С. 265-268.

11. Карманова М. Б. Максимальные поверхности-графики на 4-мерных 2-ступенчатых сублоренцевых структурах// Сиб. мат. ж. - 2016. - 57, № 2. - С. 350-362.

12. Кобалси Ш., Номидзу К. Основы дифференциальной геометрии. - М.: Наука, 1981.

13. Лаптев $Г$. Ф. К инвариантной аналитической теории дифференцируемых отображений// Тр. геом. семин. ВИНИТИ - 1974. - 6. - С. 37-42.

14. Матиева Г. Геометрия частичных отображений, сетей и распределений евклидова пространства. Ош, 2003.

15. Матиева Г., Ташпулатов А. Б. Двойные линии вырожденного частичного отображения евклидова пространства, порождаемого заданной циклической сетью Френе// Вестн. Кыргыз.-Росс. славянск. ун-та. $-2010 .-10$, № 9. - С. 20-23.

16. Матиева Г., Мустапакулова Ч. А., Папиева Т. М. О двойных линиях одного вырожденного частичного отображения евклидова пространства// в кн.: Перспективы развития фундаментальных наук (Вайтулевич Е. А., ред.). - Томск, 2014. - С. 633-635.

17. Матиева Г., Абдуллаева Ч. Х., Курбанбаева Н. Н. О квазидвойных линиях одного частичного отображения, порождаемого заданным семейством гладких линий// Ceteric Paribus. - 2016. - № 4. C. $6-13$.

18. Матиева Г., Абдуллаева Ч. Х., Папиева Т. М. О неподвижных прямых одного частичного отображения евклидова пространства $E_{5} / /$ в кн.: Наука, новые технологии и инновации Кыргызстана. Бишкек, 2017. - С. 148-151.

19. Рыжков В. В. Дифференциальная геометрия точечных соответствий между пространствами// в кн.: Итоги науки. Алгебра. Топология. Геометрия. - М.: Ин-т научн. и техн. информ. АН СССР, 1970. - C. $153-174$.

20. Рылов A. A. К геометрии отображения римановых многообразий пониженного ранга// Диффер. геом. многообр. фигур. - 1990. - 21. - С. 90-93.

21. Синюков Н. С. Геодезические отображения римановых пространств. - М.: Наука, 1970.

22. Степанов C. E. О геометрии проективных субмерсий римановых многообразий// Изв. вузов. Мат. 1999. - № 9. - C. 48-54. 
23. Abe N., Hasegawa K. An affine submersion with horizontal distribution and its applications// Differ. Geom. Appl. - 2001. - 14, № 3. - P. 235-250.

24. Chen B. Y. Geometry of Submanifolds. - New York: Marcel Dekker, 1973.

25. Chiang Y. J. f-Biharmonic maps between Riemannian manifolds// J. Geom. Symmetry Phys. — 2012. — 27. - P. 45-58.

26. Craveiro de Carvalho F. J., Robertson S. A. Graphs and their parallel groups// Rend. Circ. Mat. Palermo. Ser. 2. - 1999. - 48, № 1. - P. 65-70.

27. Mikes J. et al. Differential Geometry of Special Mappings. - Olomouc: Palacky Univ., 2015.

28. Dillen F., Verbouwe G., Vrancken L. Cubic form geometry for immersions in centro-affine and graph hypersurfaces// Res. Math. - 2003. - 43, № 1-2. - P. 88-95.

29. Eells J. Minimal graphs// Manuscr. Maths — 1979. — 28, № 1-3. — P. 101-108.

30. Falcitelli M., Ianus S., Pastore A. M. Riemannian Submersions and Related Topics. - Singapore: World Scientific, 2004.

31. Garcia-Rio E., Kupeli D. N. Semi-Riemannian Maps and Their Applications. - Dordrecht: Kluwer Academic, 1999.

32. Lee K. W., Lee Y. I. Mean curvature flow of the graphs of maps between compact manifolds// Trans. Am. Math. Soc. - 2011. - 363, № 11. - P. 5745-5759.

33. $L u W$. J. On $f$-bi-harmonic maps and bi-f-harmonic maps between Riemannian manifolds// Sci. China Math. - 2015. - 58, № 7. - P. 1483-1498.

34. Nakauchi N., Urakawa H., Gudmundsson S. Biharmonic maps into a Riemannian manifold of non-positive curvature// Geom. Dedic. - 2014. - 169. - P. 263-272.

35. Nore T. Second fundamental form of a map// Ann. Mat. Pura Appl. — 1987. — 4, № 146. — P. 281-310.

36. Ou Y. L. Lu S. Biharmonic maps in two dimensions// Ann. Mat. Pura Appl. — 2013. — 192, № 1. — P. 127-144.

37. Ou Y. L. f-Harmonic morphisms between Riemannian manifolds// Chin. Ann. Math. D. — 2014. - 35, № 2. - P. 225-236.

38. Pipoli G. Mean curvature flow and Riemannian submersion// Geom. Dedic. — 2016. — 184, № 1 . — P. $67-81$.

39. Savas-Halilaj A., Smoczyk K. Homotopy of area decreasing maps by mean curvature flow// Adv. Math. 2014. - 255. - P. 455-473.

40. Stepanov S. E. On the global theory of some classes of mappings// Ann. Glob. Anal. Geom. - 1995. 13, № 3. - P. 239-249.

41. Stepanov S., Tsyganok I. Vanishing theorems for harmonic mappings into non-negatively curved manifolds and their applications// Manuscr. Math. — 2017. — 154, № 1-2. — P. 79-90.

42. Toth G. Harmonic and Minimal Maps. - Ellis Horwood, 1984.

43. Urakawa H. The geometry of biharmonic maps// Proc. Int. Conf. "Harmonic Maps and Differential Geometry" (Cagiary, Sept. 7-10, 2009). — Providence, Rhode Island: Am. Math. Soc., 2011. — P. 159-175.

44. Urakawa $H$. Biharmonic maps into symmetric spaces and integrable systems// Hokkaido Math. J. — 2014. - 43, № 1. - P. 105-136.

45. Vilms J. Totally geodesic maps// J. Differ. Geom. — 1970. — 4, № 1. — P. 73-79.

46. Wan T. Y. H. Stability of minimal graphs in product of surfaces// Proc. Pacific Rim. Geom. Conf. "Geometry from the Pacific Rim" (Singapore, December 12-17, 1994). — Berlin-New York: de Gruyter, 1997. - P. 395-401.

47. Yano K., Ishihara S. Harmonic and relatively affine mappings// J. Differ. Geom. — 1975. — 10. — P. 501509.

Рылов Александр Аркадьевич

Финансовый университет при Правительстве Российской Федерации, Москва

E-mail: alexander_rylov@mail.ru 